\title{
Acute pancreatitis associated with pegylated interferon-alpha-2a therapy in chronic hepatitis C
}

\author{
Jong Wook Choi, June Sung Lee, Woo Hyun Paik, Tae Jun Song, Jung Wook Kim, Won Ki Bae, Kyung-Ah Kim, \\ and Jung Gon Kim
}

Department of Internal Medicine, Inje University Ilsan Paik Hospital, Goyang, Korea

Chronic hepatitis $\mathrm{C}$ virus (HCV) infection is a major cause of liver cirrhosis and hepatocellular carcinoma. Combination therapy of pegylated interferon-alpha (PEG-IFN-a) and ribavirin (RBV) is a current standard treatment for chronic $\mathrm{HCV}$ infection in Korea, which has considerable adverse effects. Acute pancreatitis is a rare complication of PEG-IFN-a administration. We report a case of a 62-year-old female who experienced acute pancreatitis after 4 weeks of PEG-IFN$a-2 a$ and RBV combination therapy for chronic HCV infection. The main cause of the acute pancreatitis in this case was probably PEG-IFN- $a$ rather than RBV for several reasons. A few cases have been reported in which acute pancreatitis occurred during treatment with PEG-IFN- $a-2 b$. This is the first report of acute pancreatitis associated with PEG-IFN- $a-2 a$ in Korea. (Clin Mol Hepatol 2016;22:168-171)

Keywords: Pegylated interferon alpha-2a; Pancreatitis; Hepatitis C virus

\section{INTRODUCTION}

Hepatitis C virus (HCV) infection is a major cause of chronic liver disease worldwide, and about $3 \%$ of the global population is estimated to have chronic HCV infection.' Chronic HCV infection can progress to liver cirrhosis in about $15-56 \%$ over periods of $20-25$ years. Annual incidence of hepatic decompensation is $1-4 \%$, that of hepatocellular carcinoma is $1-4.9 \%$ and overall mortality is $2-4 \%$ in patients with HCV-related cirrhosis. Successful HCV eradication indicated as a sustained virological response can prevent the development of cirrhosis and HCC, and reduce HCV-related mortality. ${ }^{2}$ Therefore, all HCV-infected patients without contraindication to treatment are considered as subjects for treatment. A combined therapy of pegylated interferon-alpha (PEG-IFN- $\alpha$ ) and ribavirin (RBV) is a current standard treatment regimen in Korea. ${ }^{3}$

Interferon-alpha (IFN- $\mathrm{a}$ ) is an immunomodulator and may accompany a diversity of side effects, such as flu-like symptoms, diarrhea, rash, nausea, irritability, neutropenia and thyroid dysfuction. ${ }^{4}$ Acute pancreatitis is a rare complication of the therapy.

In a retrospective study that analyzed 1,706 chronic $C$ hepatitis patients treated with IFN- $\alpha$ and RBV, seven patients $(0.4 \%)$ developed acute pancreatitis. ${ }^{5}$ However, this study analyzed the patients who had received treatment with conventional IFN- $a$, not PEG-IFN-a. We report a case of acute pancreatitis that occurred in a 62-year-old female during the treatment with PEG-IFN- $a$-2a and RBV for chronic hepatitis C. PEG-IFN- $a$ has advantages over conventional IFN- $a$ which include fewer complications, better compliance, and better therapeutic effect. It has been reported

\section{Abbreviations:}

ALP, alkaline phosphatase; ALT, alanine aminotransferase; AST, aspartate aminotransferase; CRP, C-reactive protein; CT, computed tomography; HCV, hepatitis C virus; PEG-IFN-a, pegylated interferon alpha; RBV, ribavirin; r-GT, gamma-glutamyl transpeptidase

\section{Corresponding author: June Sung Lee}

Division of Gastroenterology, Department of Internal Medicine, Inje University Ilsan Paik Hospital, 170 Juhwa-ro, Ilsanseo-gu, Goyang 10380, Korea

Tel: +82-31-910-7211, Fax: +82-31-910-7219

E-mail: jsleemd@paik.ac.kr 
that PEG-IFN- $a-2 b$ caused acute pancreatitis in a few cases. ${ }^{6,7}$ However, this is the first case of acute pancreatitis induced by PEG-IFN- $\boldsymbol{\alpha}$-2a for chronic hepatitis C treatment in Korea.

\section{CASE REPORT}

A 62-year-old female was diagnosed as chronic hepatitis $C$ and had been treated with PEG-IFN- $\mathbf{a}-2 \mathrm{a} 180$ mcg per week and RBV $1,000 \mathrm{mg}$ per day. She was admitted for severe epigastric pain on the fourth day after 4th injection of PEG-IFN- $\mathbf{a}-2 \mathrm{a}$. She had radiating pain in the back area accompanied by nausea, vomiting, and fever. On physical exam, there was tenderness in epigastric area.

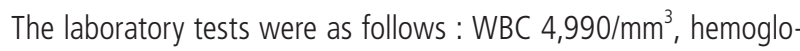
bin $12.0 \mathrm{~g} / \mathrm{dL}$, platelet $99,000 / \mathrm{mm}^{3}, \mathrm{CRP}<1 \mathrm{mg} / \mathrm{L}$, AST/ALT 38/38 IU/L, ALP/r-GT 109/19 IU/L, total bilirubin $0.9 \mathrm{mg} / \mathrm{dL}$, serum amylase 1,057 IU/L, serum lipase 1,840 IU/L, triglyceride 171 $\mathrm{mg} / \mathrm{dL}$ (30-200 mg/dL), calcium $0.96 \mathrm{mmol} / \mathrm{L}$ (0.9-1.3 mmol/L). Abdominal computed tomography (CT) scan showed diffuse swelling of the pancreas, peripancreatic fat stranding, and peripancreatic fluid collection, which were compatible with the findings of acute pancreatitis (Fig. 1A).

It was the first episode of acute pancreatitis for the patients. She had no other risk factors of acute pancreatitis. She was a nondrinker. There was no evidence of biliary stone, dilatation of the bile duct, or autoimmune pancreatitis on the ultrasound and CT scan. The levels of triglyceride and calcium were normal. She was treated for acute pancreatitis conservatively. Her abdominal pain improved after fasting, and the amylase and lipase levels decreased gradually. We began to treat her again with the PEG-IFNa-2a and RBV on the third day of hospitalization, and on the fifth day of hospitalization, her abdominal pain.

Fever relapsed, and her amylase and lipase levels increased again (248 IU/L, $585 \mathrm{IU} / \mathrm{L}$ ) (Fig. 2). Since PEG-IFN- $\boldsymbol{\alpha}-2 \mathrm{a}$ and RBV treatment seemed to be the cause of relapsed pancreatitis, the combination therapy of PEG-IFN- $-\mathrm{a}-2 \mathrm{a}$ and RBV was stopped on the sixth day of hospitalization. On the 12th day of hospitalization, abdominal pain was improved and diet was started. After 19 days of conservative management, she was discharged from the hospital with the resolution of pancreatitis (Fig. 1B). At the time of discharge, amylase and lipase levels were $133 \mathrm{IU} / \mathrm{L}$ and $156 \mathrm{IU} / \mathrm{L}$, respectively. For seven months thereafter, there was no evidence of recurrent pancreatitis.

\section{DISCUSSION}

In this case, pancreatitis was diagnosed based on its typical symptoms, elevated levels of amylase and lipase, and a CT findings corresponding with acute pancreatitis. The patient had no history of alcohol intake, no evidence of biliary stones and autoimmune pancreatitis on the abdominal ultrasound and CT scan, no anatomical abnormality, and no abnormal liver function test. Her calcium and triglyceride levels were also normal, and she did not take any special medication. Based on the fact that she had no obvious cause of pancreatitis except for HCV treatment-pan-
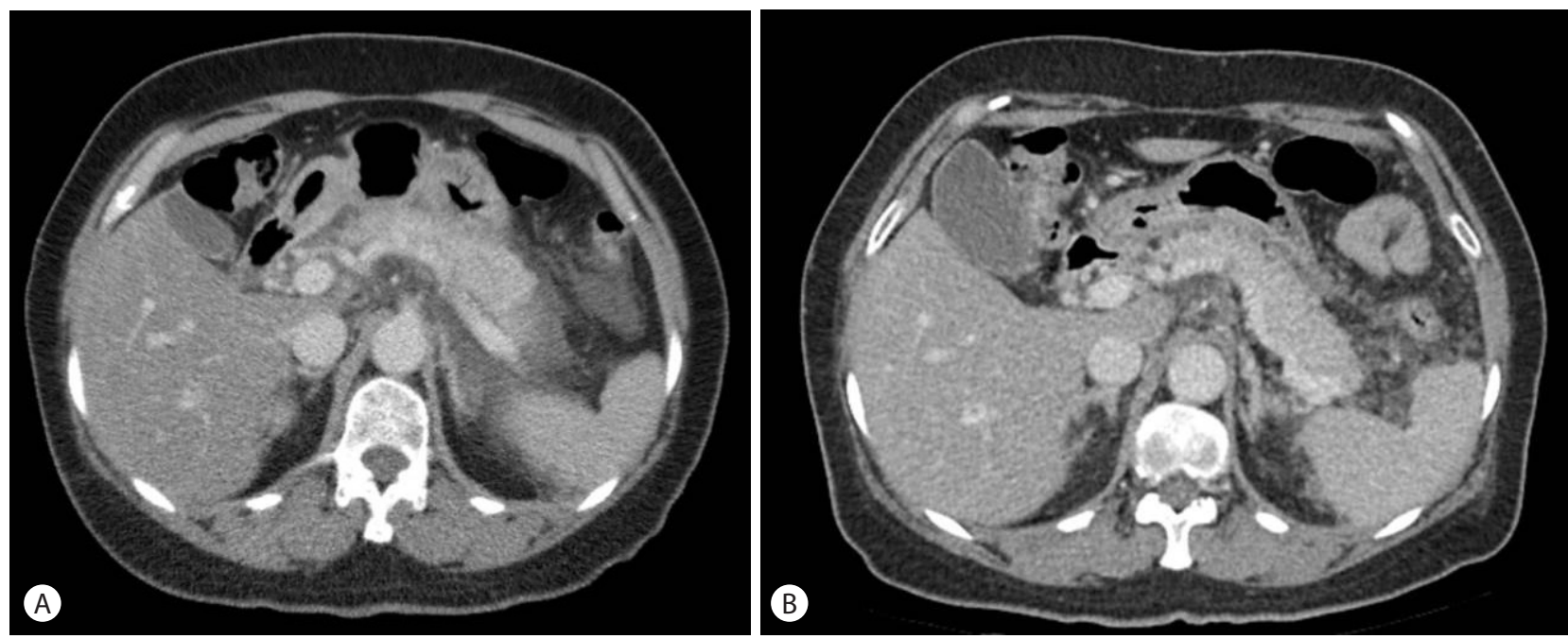

Figure 1. Enhanced computed tomography (CT) scan shows diffuse swelling of the pancreas, peripancreatic fat stranding, and fluid collection, which represents acute pancreatitis (A). Peripancreatic fat stranding and fluid collection were improved after 10 days of hospitalization (B). There was no evidence of autoimmune pancreatitis, biliary stone, or dilatation of the bile duct. 


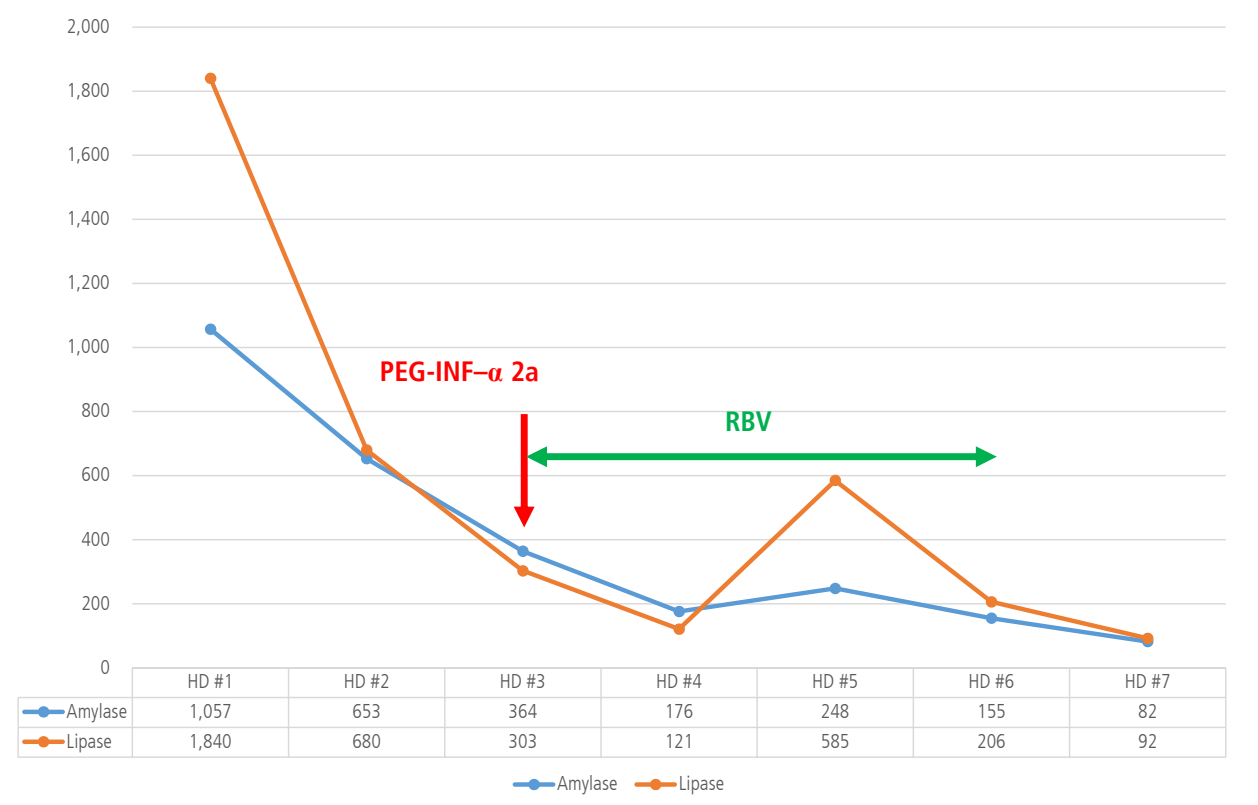

Figure 2. Variations of amylase and lipase levels during hospitalization. Her abdominal pain improved and the amylase and lipase levels decreased, and she was treated again with PEG-IFN- $\alpha-2 a$ and RBV on the third day of hospitalization. RBV was administered until the sixth day of hospitalization, when the amylase and lipase levels decreased again. PEG-IFN-a, pegylated interferon alpha; RBV, ribavirin; $H D$, hospital day. creatitis was the first event after HCV treatment, aggravated after readministration of PEG-IFN- $\alpha$ and RBV, and there was no recurrence after stopping HCV treatment-she was highly likely to have pancreatitis by PEG-IFN- $\alpha$ and RBV. Her Naranjo algorithm score, which represents an association between the adverse event and drug, was nine, suggesting her condition was closely related to the antiviral treatment. ${ }^{8}$ Because there wasn't any evidence of autoimmune pancreatitis on abdominal-pelvic $\mathrm{CT}$, we didn't investigate the serum IgG level.

Both of IFN- $\alpha$ and RBV are a potential cause of acute pancreatitis. IFN can cause pancreatitis through several potential mechanism. IFN may trigger hypertriglyceridemia, which may result in pancreatitis. IFN may also stimulate the immune system, leading to autoimmune destruction of the pancreas. As side effects of IFN, diverse kinds of autoimmune diseases, including thyroid diseases, systemic lupus erythematosus, type 1 diabetes, and asthma, may occur. ${ }^{4,9}$ The patient had normal result of thyroid function test and triglyceride level performed before and after the occurrence of pancreatitis. RBV, nucleoside analogue, is also known to exert immunomodulatory effects. PEG-IFN-a is probably regarded as the main cause of acute pancreatitis in this case because pancreatitis improved even when the patient took RBV until the 6th day of hospitalization. Moreover, based on the published literature, the relationship between RBV and pancreatitis is not definite and IFN- $\boldsymbol{\alpha}$ is more likely than RBV to cause acute pancreatitis.

PEG-IFN refers to IFN combined with a polyethylene glycol polymer chain.
Through this pegylation, PEG-IFN has longer half-life, lower dose requirement, longer interval of drug administrations, and decreasing toxicity of medication than IFN. There are two kinds of PEG-IFN-a: IFN- $a$ 2a and 2b. PEG-IFN- $a-2 a$ is the combination of $40 \mathrm{kD}$ polyethylene glycol with IFN, while PEG-IFN- $a-2 b$ is the combination of $12 \mathrm{kD}$ polyethylene glycol with IFN. ${ }^{10}$ Such differences in moiety and position of PEG cause crucial differences between the two drugs in pharmacodynamic and pharmacokinetic properties. PEG-IFN- $\mathbf{a}-2 \mathrm{a}$ is absorbed at a more constant rate than PEG-IFN- $a-2 b$, reaching a maximum concentration later than PEG-IFN- $a-2 b$. Moreover, whereas the maximum concentration of PEG-IFN- $a-2 b$ is maintained for 48 to 72 hours, that of PEG-IFN$a-2 a$ is maintained for 168 hours. Although treatment effects and safety of the two drugs are still controversial, in a study that randomly assigned 3,070 patients who had HCV genotype 1 infection treated with three regimens-standard dose PEG-IFN- $a-2 b$ or low dose PEG-IFN- $a-2 b$, or PEG-IFN- $a$-2a plus ribavirin (RBV) - , the rates of sustained virologic response, safety and adverse event did not differ significantly between the two available PEG-IFN- $\alpha$ and RBV regimens."

The cases of acute pancreatitis that have occurred worldwide during treatment of HCV with PEG-IFN- $a$ and RBV are rare, but a few cases have been reported. In Korea, two cases were reported in 2013, but they were acute pancreatitis that took place during treatment with PEG-IFN- $a-2 b$ and RBV. ${ }^{12}$ This case is meaningful in that it is the first case of acute pancreatitis in korea that happened during treatment with PEG-IFN- $a-2 a$ and RBV. 
Currently, the treatment of HCV is stopped in $10-20 \%$ of patients due to serious side effects from a combined therapy of PEGIFN- $\alpha$ and RBV; the dose is reduced in additional $20 \%$ to $30 \%$ of patients. ${ }^{4,13,14}$ When adverse events such as leukopenia, thrombocytopenia, neuropsychiatric abnormality, and autoimmune diseases occur, drug administration should be modified or suspended. When pancreatitis by IFN- $a$ and RBV is suspected, as in this case, drug administration should be discontinued.

In conclusion, acute pancreatitis may occur during the combination therapy of PEG-IFN- $\alpha$-2a and RBV for chronic hepatitis $C$, and it is mandatory to stop administration of the medication if pancreatitis caused by PEG-IFN- $a-2 a$ and RBV is suspected.

\section{Conflicts of Interest}

The authors have no conflicts to disclose.

\section{REFERENCES}

1. Mohd Hanafiah K, Groeger J, Flaxman AD, Wiersma ST. Global epidemiology of hepatitis $C$ virus infection: new estimates of agespecific antibody to HCV seroprevalence. Hepatology 2013;57:13331342.

2. Gomez EV, Rodriguez YS, Bertot LC, Gonzalez AT, Perez YM, Soler $E A$, et al. The natural history of compensated HCV-related cirrhosis: a prospective long-term study. J Hepatol 2013;58:434-444.

3. Ghany MG, Strader DB, Thomas DL, Seeff LB. Diagnosis, management, and treatment of hepatitis C: an update. Hepatology 2009;49:1335-1374.

4. Manns MP, Wedemeyer $H$, Cornberg M. Treating viral hepatitis C: efficacy, side effects, and complications. Gut 2006;55:1350-1359.

5. Chaudhari S, Park J, Anand BS, Pimstone NR, Dieterich DT, Batash $S$, et al. Acute pancreatitis associated with interferon and ribavirin therapy in patients with chronic hepatitis C. Dig Dis Sci 2004;49:1000-1006.

6. Tahan V, Tahan G, Dane F, Uraz S, Yardim M. Acute pancreatitis attributed to the use of pegylated interferon in a patient with chronic hepatitis C. J Gastrointestin Liver Dis 2007;16:224-225.

7. Ando K, Kim SR, Imoto S, Nakajima T, Mita K, Fukuda K, et al. Acute Pancreatitis Associated with Pegylated Interferon and Ribavirin Treatment of Chronic Hepatitis C, Genotype 1b with High Viral Load. Case Rep Gastroenterol 2009;3:372-376.

8. Naranjo CA, Busto U, Sellers EM, P Sandor MD, Ruiz I, Roberts EA, et al. A method for estimating the probability of adverse drug reactions. Clin Pharmacol Ther 1981;30:239-245.

9. Tomer Y, Blackard JT, Akeno N. Interferon alpha treatment and thyroid dysfunction. Endocrinol Metab Clin North Am 2007;36:10511066.

10. Zeuzem S, Welsch C, Herrmann E. Pharmacokinetics of peginterferons. Semin Liver Dis 2003;23:23-28.

11. McHutchison JG, Lawitz EJ, Shiffman ML, Muir AJ, Galler GW, McCone J, et al. Peginterferon alfa-2b or alfa-2a with ribavirin for treatment of hepatitis C infection. N Engl J Med 2009;361:580-593.

12. An S, Gu YH, Gu C, Ji HR, Jung JK. 2 Case report of acute pancreatitis during the treatment of interferon and ribavirin in chronic hepatitis C. [Abstract]. Korean J med 2013;85(cho1):91-92.

13. Fried MW. Side effects of therapy of hepatitis $C$ and their management. Hepatology 2002;36(5 Suppl 1):S237-S244.

14. Russo MW, Fried MW. Side effects of therapy for chronic hepatitis $C$. Gastroenterology 2003;124:1711-1719. 